\title{
Seleção e classificação multivariada de modelos não lineares para frangos de corte
}

\author{
[Selection and classification of multivariate nonlinear models for broilers] \\ R.C. Veloso ${ }^{1}$, L.K. Winkelstroter ${ }^{2}$, M.T.P. Silva ${ }^{3}$, A.V. Pires ${ }^{3}$, R.A. Torres Filho ${ }^{4}$, S.R.F. Pinheiro ${ }^{3}$, \\ L.S. Costa ${ }^{3}$, J.M. Amaral ${ }^{3}$ \\ ${ }^{1}$ Universidade Federal de Viçosa - Viçosa, MG \\ ${ }^{2}$ Universidade Federal de Minas Gerais - Belo Horizonte, MG \\ ${ }^{3}$ Universidade Federal dos Vales do Jequitinhonha e Mucuri - Diamantina, MG \\ ${ }^{4}$ Universidade Federal Fluminense - Niterói, RJ
}

\begin{abstract}
RESUMO
Objetivou-se com este estudo utilizar a técnica de análise de agrupamento para classificar modelos de regressão não lineares usados para descrever a curva de crescimento de frangos de corte, levando em consideração os resultados de diferentes avaliadores de qualidade de ajuste. Para tanto, utilizaram-se dados de peso corporal e idade dos seguintes grupos genéticos de frangos de corte: Cobb500, Hubbard Flex e Ross308, de ambos os sexos, constituindo, assim, seis classes. Foram ajustados 10 modelos não lineares, cuja qualidade de ajuste foi medida pelo coeficiente de determinação ajustado, pelos critérios de informação de Akaike e bayesiano, pelo quadrado médio do erro e pelo índice assintótico. A análise de agrupamento indicou os modelos logístico, Michaelis-Menten, Michaelis-Menten modificado e von Bertalanffy como os mais adequados à descrição das curvas de crescimento das seis classes estudadas.
\end{abstract}

Palavras-chave: agrupamento, curva de crescimento, idade, peso corporal

\begin{abstract}
The aim of this study was to classify non-linear models used to describe the growth curve of broilers using the cluster analysis technique, taking into account the results of different measures of quality adjustment regression. For this purpose, we used data of body weight and age the following genetic groups of broilers: Cobb500, Hubbard Flex and Ross308, of both sexes, thus constituting six classes. Ten non-linear models were fitted, the quality of fit was measured by the adjusted coefficient of determination, Akaike information criteria and Bayesian, mean square error and index asymptotic. Cluster analysis indicated the Logistico, Michaelis Menten, Michaelis Menten Modificado and von Bertalanffy models as the most appropriate description of the growth curves for the six classes studied.
\end{abstract}

Keywords: age, body weight, cluster, growth curve

\section{INTRODUÇÃO}

O crescimento é uma propriedade fundamental do sistema biológico, podendo ser definido como um aumento no tamanho do corpo por unidade de tempo (Schulze et al., 2001). Nesse sentido, o estudo de curvas de crescimento de modelagem de animais é necessário para otimizar a gestão e a eficiência da produção animal (Köhn et al., 2007).

Recebido em 7 de agosto de 2014

Aceito em 16 de setembro de 2015

E-mail: velosozootecnista@yahoo.com.br
Os modelos não lineares têm sido propostos para descrição da curva de crescimento dos indivíduos, pois apresentam parâmetros com interpretação biológica e, assim, possibilitam maior entendimento do processo de crescimento dos animais. Porém, na maioria das vezes, a escolha dos melhores modelos não é tão simples. Por causa do grande número de avaliadores de qualidade de ajustes existentes na literatura, muitas vezes há discordância entre eles para a 
definição do melhor modelo a ser utilizado para descrever o crescimento dos animais. Assim, uma análise que considere simultaneamente todos os avaliadores de qualidade é necessária para estabelecer qual modelo melhor se ajusta aos dados de crescimento.

Dessa maneira, uma análise complementar que pode ser útil seria a análise de agrupamento, a fim de agrupar modelos cujos resultados dos avaliadores de qualidade de ajuste sejam similares dentro de cada população. Essa técnica de agrupamento tem sido proposta por diversos autores (Maia et al., 2009; Silva et al., 2011; Silveira et al., 2011; Silveira et al., 2012).

Nesse contexto, objetivou-se com este trabalho utilizar a análise de agrupamento para classificar modelos não lineares, usados para descrever a curva de crescimento de três genótipos de frangos de corte de ambos os sexos.

\section{MATERIAL E MÉTODOS}

Os dados utilizados são provenientes de um experimento em um delineamento inteiramente ao acaso, realizado com frangos de corte, que foi conduzido no Departamento de Zootecnia da UFVJM, situado no Campus JK, em DiamantinaMG, durante o período de 31 de outubro a 19 de dezembro de 2011, aprovado pela Comissão de Ética no Uso de Animais da UFVJM (Protocolo $\left.n^{\circ} 015 / 10\right)$.

Utilizaram-se dados de pesos corporais individuais dos frangos de corte medidos a um, sete, 14, 21, 28, 35, 42 e 49 dias de idade, totalizando 456 aves, de três genótipos (Cobb500, Hubbard Flex e Ross308), de ambos os sexos, perfazendo um total de 152 aves por genótipo e 76 aves por sexo x genótipo, constituindo um total de seis classes (dois sexos $\mathrm{x}$ três genótipos). A ração experimental utilizada foi formulada á base de milho e farelo de soja, conforme Rostagno et al. (2011).
Os seguintes modelos de regressão não linear, descritos por Silva et al. (2011) e Silveira et al. (2011), foram ajustados aos dados de frangos de corte (Tab. 1), utilizando-se o método dos quadrados mínimos ordinários, cujas soluções foram obtidas mediante o processo iterativo de Gauss-Newton, por meio do "proc model" do SAS (SAS, 2002).

O parâmetro $\beta_{1}$ representa o peso adulto, ou peso assintótico do animal, e o parâmetro $\beta_{3}$ a taxa de maturidade, ou velocidade de crescimento, exceto para dois modelos, Michaelis-Menten modificado e Weibull, em que a taxa de maturidade é obtida como função dos demais parâmetros. Os modelos que apresentam o parâmetro $\beta_{4}$ possuem ponto de inflexão variável, cuja localização é determinada pelo parâmetro em questão, e os demais modelos ou apresentam o ponto de inflexão fixo ou não o possuem, como é o caso do modelo MichaelisMenten. De forma geral, não há uma interpretação prática para o parâmetro $\beta_{2}$ (constante de integração), $y_{i}$ representa a observação da variável dependente designada de variável resposta, e $\mathrm{x}_{\mathrm{i}}$ a variável independente designada de variável explicativa.

Foram utilizados cinco avaliadores da qualidade de ajuste: coeficiente de determinação ajustado $\left(\mathrm{R}^{2} \mathrm{aj}\right)$, quadrado médio do erro (QME), critério de informação de Akaike (AIC) e critério de informação bayesiano (BIC), proposto por Silva et al. (2011), e índice assintótico (IA), descrito por Ratkowsky (1990) e utilizado por Araújo et al. (2012) e Drumond et al. (2013), o qual combina os seguintes critérios: desvio padrão assintótico (DPA), desvio médio absoluto (DMA) e coeficiente de determinação $\left(\mathrm{R}^{2}\right)$, atribuindo valor 100 para a maior estimativa de cada critério, sendo os demais ponderados em relação a este, assim: $\mathrm{IA}=(\mathrm{DPA}+\mathrm{DMA})-\mathrm{R}^{2}$, em que quanto menor o índice, melhor o ajuste do modelo. 
Tabela 1. Modelos de regressão não linear para descrever curvas de crescimento dos frangos de corte

\begin{tabular}{|c|c|}
\hline Modelo & Função \\
\hline A- Meloun I & $y_{i}=\beta_{1}-\beta_{2} e^{\left(-\beta_{3} x_{1}\right)}+e_{i}$ \\
\hline B- Meloun II & $y_{i}=\beta_{1}-e^{\left(-\beta_{2}-\beta_{3} x_{1}\right)}+e_{i}$ \\
\hline C- Michaelis-Menten & $y_{i}=\frac{\beta_{1} x_{i}}{x_{i}+\beta_{2}}+e_{i}$ \\
\hline D- Weibull & $y_{i}=\beta_{1}-\beta_{2} e^{-e^{\beta_{3} x_{1}}}+e_{i}$ \\
\hline E- Logístico & $y_{i}-\frac{\beta_{1}}{\left(1+e^{\left(\beta_{2}-\beta_{3} x_{1}\right)}\right)}+e_{i}$ \\
\hline F- Gompertz & $y_{i}=\beta_{1} e^{\left(-e^{\left(\beta_{2}-\beta_{3} x_{1}\right)}\right)}+e_{i}$ \\
\hline G- Michaelis-Menten modificado & $y_{i}=\frac{\beta_{2} \beta_{3}^{\beta_{4}}+\beta_{1} x_{i}^{\beta_{4}}}{\beta_{3}^{\beta_{4}}+x_{i}^{\beta_{4}}}+e_{i}$ \\
\hline H- Von Bertalanffy & $y_{i}=\beta_{1}\left(1-\beta_{2} e^{-\beta_{3} x_{1}}\right)^{3}+e_{i}$ \\
\hline I- Richards & $y_{i}=\frac{\beta_{1}}{\left(1+e^{\left(\beta_{z}-\beta_{3} x_{1}\right)}\right)^{\frac{1}{\beta_{4}}}}+e_{i}$ \\
\hline J- Mitscherlich & $y_{i}=\beta_{1}\left(1-e^{\left(\beta_{3} \beta_{2}-\beta_{3} x_{1}\right)}\right)+e_{i}$ \\
\hline
\end{tabular}

Após a obtenção dos avaliadores de qualidade apresentados para cada um dos modelos, confeccionou-se um conjunto de dados multivariados, no qual os modelos correspondem às unidades a serem agrupadas e os avaliadores correspondem às variáveis medidas (Silva et al., 2011 e Silveira et al., 2011).

O método de agrupamento utilizado foi o método hierárquico do centroide. Nesse método, a distância entre dois grupos é definida como sendo a distância entre os vetores de médias, também chamados de centroides, dos grupos que estão sendo comparados. Segundo Mingoti (2007), é dada, então, pela distância euclidiana ao quadrado entre os centroides dos dois grupos. Nessa análise, obtiveram-se os valores do índice RMSSTD (Root Mean Square Standard Deviation, ou Raiz Quadrada do Desvio Padrão
Médio), que possibilitam identificar o número ótimo de grupos, de acordo com Cecon et al. (2008).

\section{RESULTADOS E DISCUSSÃO}

Os pesos corporais dos frangos de corte, mostrados na Tab. 2, foram semelhantes aos encontrados por Corzo et al. (2010), Tang et al. (2011), Lai et al. (2012) e Walk et al. (2012). De forma geral, observa-se que o genótipo Ross308, em ambos os sexos, apresentou maior peso corporal nas diferentes idades, e entre as fêmeas o genótipo Hubbard Flex apresentou maior peso corporal nas idades de 42 e 49 dias. Os machos do genótipo Hubbard Flex, assim como as fêmeas do genótipo Cobb500, apresentaram os menores pesos corporais. 
Apenas as equações propostas nos modelos de Richards e Mitscherlich não atingiram convergência.

Entre as que convergiram, nota-se que, de forma geral, entre os genótipos de frango de corte em ambos os sexos, conforme pode ser visualizado nas Tab. 3, 4, e 5, os melhores ajustes dos modelos foram observados para os frangos dos genótipos Hubbard Flex e Cobb500, independentemente do sexo, uma vez que foram observados maiores $\mathrm{R}_{\text {aj }}^{2}$ e menores QME, AIC, BIC e IA. Com base nessas informações, verificou-se que os dados médios dos genótipos Hubbard Flex e Cobb500 apresentam um comportamento mais característico de curva de crescimento, conforme pode ser visualizado na Tab. 2, uma vez que os modelos específicos para descrever este fenômeno se ajustaram melhor.

Tabela 2. Pesos corporais observados (em gramas) de acordo com a idade, considerando os dados médios das seis classes de frangos de corte $\mathrm{x}$ sexo estudadas

\begin{tabular}{lllllllll}
\hline \multirow{2}{*}{ Classe } & \multicolumn{7}{c}{ Idade (dia) } \\
\cline { 2 - 9 } & 1 & 7 & 14 & 21 & 28 & 35 & 42 & 49 \\
\hline Cobb500 macho & 41,00 & 129,00 & 353,52 & 761,52 & 1245,81 & 2025,69 & 2579,48 & 3367,28 \\
Hubbard Flex macho & 42,80 & 124,82 & 349,86 & 739,54 & 1175,41 & 1936,71 & 2676,31 & 3370,35 \\
Ross308 macho & 43,20 & 140,66 & 364,48 & 774,613 & 1232,21 & 1981,59 & 2649,85 & 3382,65 \\
Cobb500 fêmea & 40,08 & 119,73 & 336,48 & 694,44 & 1108,13 & 1731,75 & 2227,52 & 2777,33 \\
Hubbard Flex fêmea & 41,68 & 128,82 & 339,85 & 694,14 & 1137,57 & 1782,08 & 2368,15 & 2968,27 \\
Ross308 fêmea & 43,40 & 133,92 & 355,76 & 722,29 & 1145,35 & 1809,66 & 2296,77 & 2917,32 \\
\hline
\end{tabular}

Todos os avaliadores de qualidade, com exceção do QME e IA, apresentaram baixo coeficiente de variação. Resultados semelhantes foram observados por Silva et al. (2011). Em relação aos outros avaliadores de qualidade de ajuste (AIC e BIC), de forma geral, os modelos da "família" de Richards (Gompertz e von Bertalanffy) foram os que apresentaram os melhores avaliadores de qualidade, mostrando a superioridade desses modelos, nas condições aqui avaliadas. Resultados semelhantes foram encontrados por Drumond et al. (2013), que recomendaram a utilização dos modelos Gompertz e logístico para descrição do crescimento de codornas de corte.

Pode ser observado que os maiores valores do QME foram observados para modelos com três (Meloun I e Meloun II) e quatro parâmetros (Weibull). Em bovinos de corte, Silva et al. (2011) observaram maiores valores de QME para modelos mais parametrizados, como Richards, Michaelis-Menten modificado e Weibull. Neste trabalho, o modelo Michaelis-Menten modificado apresentou baixo QME.

Quanto ao coeficiente de determinação ajustado $\left(\mathrm{R}^{2}{ }_{\text {aj }}\right)$, foi o avaliador que apresentou menores coeficientes de variação, o que permite inferir que esse avaliador realmente apresenta baixa variabilidade. Com isso, recomenda-se utilizar outros avaliadores de qualidade de ajuste, pois as diferenças entre os $\mathrm{R}_{\text {aj }}^{2}$ dos diferentes modelos usados no estudo de curvas de crescimento foram irrisórias, conforme também destacado por Oliveira et al. (2000).

O menor valor para o índice assintótico (IA) foi observado para os modelos da "família Richards" mais o modelo Michaelis-Menten modificado em todas as seis classes estudadas, indicando serem esses os modelos que mais se ajustaram aos dados, considerando que o IA é um critério que combina $\mathrm{R}^{2}$, DPA e DMA e que, portanto, em princípio, é visto como um critério mais "completo" (Araújo et al., 2012). Nesse sentido, para a escolha de modelos, pode-se recomendar o índice assintótico.

O modelo von Bertalanffy, levando em consideração o QME, o AIC e o BIC, ajustou-se melhor ao crescimento dos frangos de corte Cobb500 machos, pois os menores valores desses critérios foram observados, conforme apresentado na Tab. 3. Se a escolha do modelo for feita somente observando o IA, o modelo 
Gompertz se ajustou melhor ao crescimento dos frangos de corte Cobb500 em ambos os sexos. É interessante observar que, nas fêmeas, independentemente do critério de qualidade de ajuste a ser utilizado, o modelo Gompertz é sempre o indicado.

Tabela 3. Avaliadores da qualidade de ajuste para os modelos não lineares avaliados nos frangos de corte do genótipo Cobb500

\begin{tabular}{|c|c|c|c|c|c|}
\hline \multirow{2}{*}{ Modelo } & \multicolumn{5}{|c|}{ Avaliadores da qualidade de ajuste } \\
\hline & $\mathrm{R}_{\mathrm{aj}}^{2}$ & QME & AIC & $\mathrm{BIC}$ & IA \\
\hline \multicolumn{6}{|l|}{ Cobb500 Macho } \\
\hline A- Meloun I & 0,9972 & 1154648,00 & 10071,90 & 10076,30 & 115,79 \\
\hline B- Meloun II & 0,9966 & 1384108,00 & 10178,50 & 10182,90 & 181,58 \\
\hline C- Michaelis-Menten & 0,9985 & 122020,00 & 8729,20 & 8733,60 & 73,39 \\
\hline D- Weibull & 0,9949 & 1384108,00 & 10178,50 & 10182,90 & 1258,56 \\
\hline E- Logistico & 1,0000 & 61466,00 & 8319,60 & 8324,00 & 60,57 \\
\hline F- Gompertz & 1,0000 & 124887,00 & 8735,70 & 8740,10 & $-13,08$ \\
\hline G- Michaelis-Menten modificado & 1,0000 & 61488,00 & 8325,70 & 8347,60 & 62,35 \\
\hline H- Von Bertalanffy & 0,9985 & 61408,00 & 8319,10 & 8323,50 & 63,61 \\
\hline Média & 0,9985 & 544266,62 & 9107,27 & 9113,86 & 225,35 \\
\hline Desvio-padrão & 0,0019 & 636590,03 & 874,97 & 872,76 & 421,09 \\
\hline Coeficiente de variação (\%) & 0,1992 & 116,96 & 9,60 & 9,57 & 53,51 \\
\hline \multicolumn{6}{|l|}{ Cobb500 Fêmea } \\
\hline A- Meloun I & 0,9972 & 772411,00 & 9815,60 & 9819,90 & 109,62 \\
\hline B- Meloun II & 0,9966 & 940930,00 & 9947,30 & 9951,70 & 180,91 \\
\hline C- Michaelis-Menten & 0,9998 & 59470,00 & 8299,10 & 8303,50 & 64,91 \\
\hline D- Weibull & 0,9949 & 940930,00 & 9947,30 & 9951,70 & 1068,89 \\
\hline E- Logístico & 1,0000 & 26394,00 & 7813,70 & 7818,10 & 68,32 \\
\hline F- Gompertz & 1,0000 & 26356,00 & 7812,90 & 7817,30 & $-7,48$ \\
\hline G- Michaelis-Menten modificado & 1,0000 & 26517,40 & 7820,70 & 7842,70 & 68,47 \\
\hline H- Von Bertalanffy & 0,9999 & 26593,00 & 7818,20 & 7822,60 & 66,38 \\
\hline Média & 0,9986 & 352450,17 & 8659,35 & 8665,93 & 202,50 \\
\hline Desvio-padrão & 0,0020 & 443989,47 & 1043,81 & 1041,79 & 354,00 \\
\hline Coeficiente de variação (\%) & 0,1997 & 125,9723 & 12,05 & 12,0217 & 57,20 \\
\hline
\end{tabular}

$\mathrm{R}_{\text {aj: }}^{2}$ coeficiente de determinação ajustado; QME: quadrado médio do erro; AIC: critério de informação de Akaike; BIC: critério de informação bayesiano; IA: índice assintótico.

O modelo Logístico se ajustou melhor ao crescimento dos frangos de corte Hubbard Flex, independentemente do sexo, pois os menores valores de QME, AIC, BIC e IA foram observados, como mostra a Tab. 4, sendo o indicado para descrição da curva de crescimento do genótipo Hubbard Flex.

O modelo Logístico, levando em consideração os critérios de qualidade de ajuste QME, AIC e 
BIC, ajustou-se melhor ao crescimento dos frangos de corte Ross308 machos, pois apresentaram os menores valores, conforme mostrado na Tab. 5. Assim como observado com o genótipo Cobb500, se a escolha do modelo for feita somente observando o IA, o modelo
Gompertz se ajustou melhor ao crescimento dos frangos de corte Ross308 em ambos os sexos. Nas fêmeas, independentemente do critério de qualidade de ajuste a ser utilizado (QME, AIC, BIC e IA), o modelo Gompertz é o recomendado.

Tabela 4. Avaliadores da qualidade de ajuste para os modelos não lineares avaliados nos frangos de corte do genótipo Hubbard Flex

\begin{tabular}{|c|c|c|c|c|c|}
\hline \multirow{2}{*}{ Modelo } & \multicolumn{5}{|c|}{ Avaliadores da qualidade de ajuste } \\
\hline & $\mathrm{R}_{\mathrm{aj}}^{2}$ & QME & $\mathrm{AIC}$ & $\mathrm{BIC}$ & $\mathrm{IA}$ \\
\hline & \multicolumn{5}{|c|}{ Hubbard Flex Macho } \\
\hline A- Meloun I & 0,9971 & 1177946,00 & 10083,80 & 10088,20 & 41,39 \\
\hline B- Meloun II & 0,9966 & 1398119,00 & 10184,50 & 10188,90 & 187,53 \\
\hline C- Michaelis-Menten & 0,9998 & 128936,00 & 8762,20 & 8766,60 & 87,52 \\
\hline D- Weibull & 0,9949 & 1398119,00 & 10184,50 & 10188,90 & 1269,95 \\
\hline E- Logistico & 1,0000 & 54080,00 & 8243,10 & 8247,50 & 36,38 \\
\hline F- Gompertz & 1,0000 & 88628,00 & 8538,00 & 8542,40 & 41,51 \\
\hline G- Michaelis-Menten modificado & 1,0000 & 54572,70 & 8253,50 & 8275,50 & 51,19 \\
\hline H- Von Bertalanffy & 0,9998 & 54942,00 & 8252,60 & 8256,90 & 64,78 \\
\hline Média & 0,9986 & 544417,83 & 9062,77 & 9069,36 & 222,53 \\
\hline Desvio-padrão & 0,0020 & 650198,30 & 918,68 & 916,50 & 426,11 \\
\hline Coeficiente de variação (\%) & 0,1996 & 119,43 & 10,13 & 10,10 & 52,22 \\
\hline \multicolumn{6}{|l|}{ Hubbard Flex Fêmea } \\
\hline A- Meloun I & 0,9972 & 882455,00 & 9910,90 & 9915,30 & 39,09 \\
\hline B- Meloun II & 0,9966 & 1067240,00 & 10022,80 & 10027,20 & 187,36 \\
\hline C- Michaelis-Menten & 0,9998 & 71354,00 & 8408,20 & 8412,60 & 83,63 \\
\hline D- Weibull & 0,9949 & 1067240,00 & 10022,80 & 10027,20 & 1133,07 \\
\hline E- Logístico & 1,0000 & 26249,00 & 7810,60 & 7815,00 & 34,47 \\
\hline F- Gompertz & 1,0000 & 239513,00 & 9122,20 & 9126,60 & 56,66 \\
\hline G- Michaelis-Menten modificado & 1,0000 & 26490,60 & 7821,20 & 7843,20 & 50,34 \\
\hline H- Von Bertalanffy & 0,9998 & 57637,00 & 8281,20 & 8285,60 & 72,68 \\
\hline Média & 0,9986 & 547592,05 & 9096,57 & 9103,17 & 237,08 \\
\hline Desvio-padrão & 0,0020 & 643431,74 & 890,94 & 888,68 & 420,09 \\
\hline Coeficiente de variação (\%) & 0,1993 & 117,50 & 9,79 & 9,76 & 56,43 \\
\hline
\end{tabular}

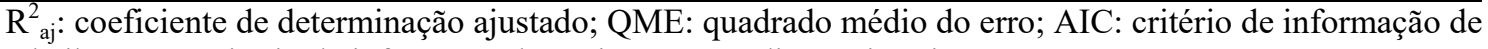
Akaike; BIC: critério de informação bayesiano; IA: índice assintótico. 
Seleção e classificação...

Tabela 5. Avaliadores da qualidade de ajuste para os modelos não lineares avaliados nos frangos de corte do genótipo Ross308

\begin{tabular}{|c|c|c|c|c|c|}
\hline \multirow{3}{*}{ Modelo } & \multicolumn{5}{|c|}{ Avaliadores da qualidade de ajuste } \\
\hline & $\mathrm{R}_{\mathrm{aj}}^{2}$ & QME & AIC & BIC & IA \\
\hline & \multicolumn{5}{|c|}{ Ross308 Macho } \\
\hline A- Meloun I & 0,9972 & 1164539,00 & 10072,80 & 10077,20 & 144,79 \\
\hline B- Meloun II & 0,9966 & 1396297,00 & 10183,70 & 10188,10 & 185,38 \\
\hline C- Michaelis-Menten & 0,9998 & 122049,00 & 8729,40 & 8733,80 & 75,72 \\
\hline D- Weibull & 0,9949 & 1396297,00 & 10183,70 & 10188,10 & 1267,0 \\
\hline E- Logístico & 1,0000 & 57359,00 & 8278,30 & 8282,70 & 77,52 \\
\hline F- Gompertz & 1,0000 & 128885,00 & 8756,00 & 8760,40 & $-10,31$ \\
\hline G- Michaelis-Menten modificado & 1,0000 & 57673,40 & 8287,50 & 8309,50 & 83,77 \\
\hline H- Von Bertalanffy & 0,9998 & 57637,00 & 8281,20 & 8285,60 & 72,68 \\
\hline Média & 0,9986 & 547592,05 & 9096,57 & 9103,17 & 237,08 \\
\hline Desvio-padrão & 0,0020 & 643431,74 & 890,94 & 888,68 & 420,09 \\
\hline Coeficiente de variação (\%) & 0,1993 & 117,50 & 9,79 & 9,76 & 56,43 \\
\hline \multicolumn{6}{|l|}{ Ross308 Fêmea } \\
\hline A- Meloun I & 0,9966 & 1022814,00 & 9963,90 & 9968,30 & 156,47 \\
\hline B- Meloun II & 0,9966 & 1022814,00 & 9963,90 & 9968,30 & 184,82 \\
\hline C- Michaelis-Menten & 0,9998 & 69470,00 & 8364,20 & 8368,60 & 74,28 \\
\hline D- Weibull & 0,9949 & 1022814,00 & 9963,90 & 9968,30 & 1110,6 \\
\hline E- Logístico & 1,0000 & 32386,00 & 7909,70 & 7914,10 & 36,97 \\
\hline F- Gompertz & 1,0000 & 32307,00 & 7908,30 & 7912,70 & 2,40 \\
\hline G- Michaelis-Menten modificado & 1,0000 & 32465,80 & 7917,00 & 7939,00 & 49,87 \\
\hline H- Von Bertalanffy & 0,9998 & 32471,00 & 7911,30 & 7915,70 & 48,35 \\
\hline Média & 0,9985 & 408442,72 & 8737,77 & 8744,37 & 207,97 \\
\hline Desvio-padrão & 0,0021 & 508902,01 & 1026,79 & 1024,80 & 369,91 \\
\hline Coeficiente de variação (\%) & 0,2062 & 124,59 & 11,75 & 11,71 & 56,22 \\
\hline
\end{tabular}

$\mathrm{R}_{\text {aj: }}^{2}$ coeficiente de determinação ajustado; QME: quadrado médio do erro; AIC: critério de informação de Akaike; BIC: critério de informação bayesiano; IA: índice assintótico.

$\mathrm{Na}$ Tab. 6, observa-se que, para todos os genótipos e ambos os sexos avaliados, o número ótimo de grupos encontrado foi quatro, ou seja, os oito modelos considerados foram condensados em apenas quatro grupos distintos, mostrando grande semelhança nos resultados para os diferentes modelos.

Percebe-se que os melhores grupos (Grupo 1) foram os que apresentaram, de forma geral, menores valores de QME, AIC, BIC e IA considerados simultaneamente. Ao se verificarem quais modelos foram predominantes na formação desses grupos, os quais contêm os melhores modelos, nota-se que houve predominância dos modelos da "família" Richards (Gompertz, logístico e von Bertalanffy) mais os modelos Michaelis-Menten e MichaelisMenten modificado. Esses modelos encontramse entre os melhores para descrever 0 crescimento dos genótipos de frangos de corte, independentemente do sexo. Nota-se que o 
modelo Gompertz encontra-se entre os melhores para descrição das seis classes de frangos de corte, devido à sua predominância em todos os grupos. Para descrição do crescimento dos machos, percebe-se que houve predominância dos modelos Michaelis-Menten, além do modelo
Gompertz, na formação dos grupos. Com relação às fêmeas, além do modelo de Gompertz, houve predominância dos modelos logístico, MichaelisMenten modificado e von Bertalanffy na formação dos grupos.

Tabela 6. Grupos com os seus respectivos modelos e médias dos avaliadores de qualidade de ajuste (AQ) para cada classe considerada

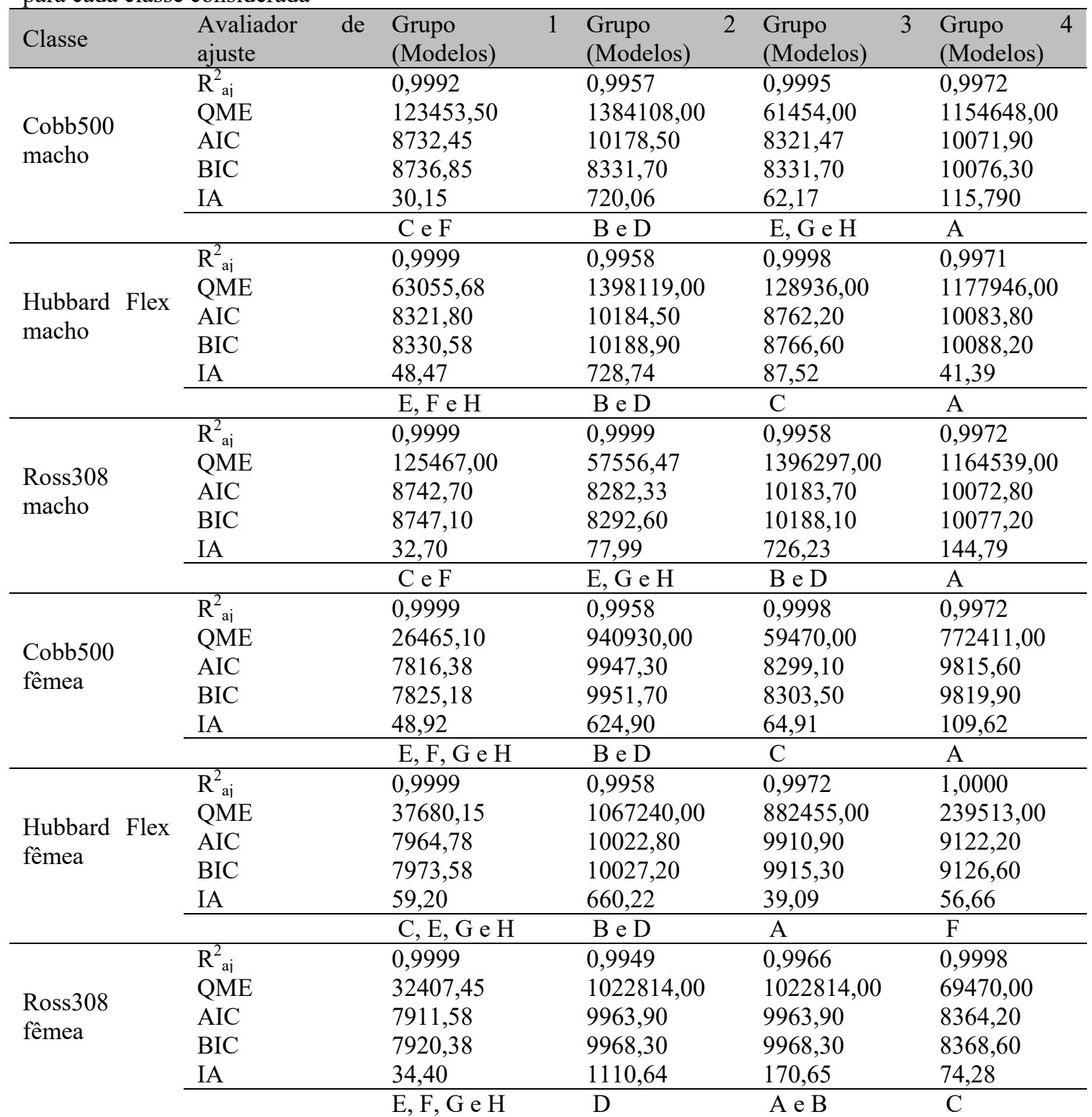

A- Meloun I; B- Meloun II; C- Michaelis-Menten; D- Weibull; E- logístico; F- Gompertz; G- Michaelis- Menten modificado; H- von Bertalanffy. 
O modelo de Gompertz tem sido recomendado por diversos autores para descrição do crescimento de frangos de corte (Wang et al., 2005; Norris et al., 2007; Rizzi et al., 2013). Entretanto, outros modelos têm sido propostos por outros autores, como Tompic et al. (2011), que, além do modelo de Gompertz, também recomendaram o modelo de Richards. Yang et al. (2006) recomendaram o modelo von Bertalanffy para descrição do crescimento de frangos de corte.

\section{CONCLUSÕES}

Os modelos Gompertz, logístico, MichaelisMenten, Michaelis-Menten modificado e von Bertalanffy, por apresentarem bons avaliadores de qualidade de ajuste, são os recomendados para descrever o crescimento dos três genótipos de frango de corte, de ambos os sexos. A análise de agrupamento proposta mostrou-se adequada para classificação dos modelos não lineares.

\section{AGRADECIMENTOS}

Os autores agradecem ao apoio financeiro recebido da Capes, do CNPq e da Fapemig.

\section{REFERÊNCIAS}

ARAÚJO, R.O.; MARCONDES, C.R.; DAMÉ, M.C.F. et al. Classical nonlinear models to describe the growth curve for Murrah buffalo breed. Ciênc. Rural, v.42, p.520-525, 2012.

CECON, P.R.; SILVA, F.F.; FERREIRA, A. et al. Análise de medidas repetidas na avaliação de clones de café 'Conilon'. Pesqui. Agropecu. Bras., v.43, p.1171-1176, 2008.

CORZO, A.; SCHILLING, M. W.; LOAR II, R. E. et al. Responses of Cobb x Cobb 500 broilers dietary amino acid density regimens. J. Appl. Poult. Res.. v.19, p.227-236, 2010.

DRUMOND, E.S.C.; GONÇALVES, F.M.; VELOSO, R.C. et al. Curvas de crescimento para codornas de corte. Ciênc. Rural, v.43, p.1872-1877, 2013.

KÖHN, F.; SHARIFI A.R.; SIMIANER, H. Modeling the growth of the Gottingen minipig. $J$. Anim. Sci., v.85, p.84-92, 2007.
LAI, H.T.L.; NIEUWLAND, M.G.; AARNINK, A.J. et al. Effects of 2 size classes of intratracheally administered airborne dust particles on primary and secondary specific antibody responses and body weight gain of broilers: A pilot study on the effects of naturally occurring dust. Poult. Sci., v.91, p.604-615, 2012.

MAIA, E; SQUEIRA, D.L.; SILVA, F.F. et al. Método de comparação de modelos de regressão não-lineares em bananeiras. Ciênc. Rural, v.39, p.1380-1386, 2009.

MINGOTI, S.A. Análise de dados através de métodos de estatística multivariada: uma abordagem aplicada. Belo Horizonte: UFMG, 2007. 295p.

NORRIS, D.; NGAMBI, J.W.; BENYI, K. et al. Analysis of growth curves of indigenous male Venda and Naked Neck chickens. S. Afr. J. Anim. Sci., v.37, p.21-26, 2007.

OLIVEIRA, H.N.; LOBO, R.B.; PEREIRA, C.S. Comparação de modelos não-lineares para descrever o crescimento de fêmeas da raça Guzerá. Pesqui. Agropecu. Bras., v.35, p.18431851, 2000.

RATKOWSKY, D.A. Handbook of nonlinear regression models. New York: Marcel Dekker, 1990. 241p.

RIZZI, C.; CONTIERO, B.; CASSANDRO, M. Growth patterns of Italian local chicken population. Poult. Sci., v.92, p.2226-2235, 2013.

ROSTAGNO, H.S. et al. Tabelas brasileiras para aves e suínos: composição de alimentos e exigências nutricionais. 3.ed. Viçosa: Universidade Federal de Viçosa, 2011. 252p.

SAS user's guide. Version 9.1. Cary: Statistical Analysis Systems, 2002. v.2, 1052p.

SCHULZE, V.; ROHE, R.; LOOFT, H.; KALM, E. Genetic analysis of the course of individual growth and feed intake of group-penned performance-tested boars. Arch. Tierzucht, v.44, p.139-156, 2001.

SILVA, N.A.M.; LANA, A.M.Q.; SILVA, F.F. et al. Seleção e classificação multivariada de modelos de crescimento não lineares para bovinos Nelore. Arq. Bras. Med. Vet. Zootec., v.63, p.364-371, 2011. 
SILVEIRA, F.G.; SILVA, F.F.; CARNEIRO, P.L.S. et al. Análise de agrupamento na seleção de modelos de regressão não-lineares para curvas de crescimento de ovinos cruzados. Rev. Ciênc. Rural, v.41, p.692-698, 2011.

SILVEIRA, F.G.; SILVA, F.F.; CARNEIRO, P.L.S.; MALHADO, C.H.M. Classificação multivariada de modelos de crescimento para grupos genéticos de ovinos de corte. Rev. Bras. Saúde Prod. Anim., v.13, p.62-73, 2012.

TANG, S.C.; ZULKIFLI, I.; EBRAHIMI, M. et al. Effects of feeding different levels of corn dried distillers grains with solubles on growth performance, carcass yeld and meat fatty acid composition in broiler chickens. Int. J. Anim. Vet. Adv., v.3, p.205-211, 2011.
TOMPIC, T.; DOBSA, J.; LEGEN, S. et al. Modeling the growth pattern of in-season and off-season Ross 308 broiler breeder flocks. Poult Sci., v.90, p.2879-2887, 2011.

WALK, C. L.; ADDO-CHIDIE, E.K.; BEDFORD, M.R.; ADEOLA, O. Evaluation of a highly soluble calcium source and phytase in the diets of broiler chickens. Poult. Sci., v.91, p.2255-2263, 2012.

WANG, C. F.; ZHANG, L.; LI, J.Y.; WU, C.X. Analysis of body conformation and fitting growth model in Tibetan chicken raised in plain. Sci. Agric. Sinica, v.38, p.1065-1068, 2005.

YANG, Y.; MEKKI, D.M.; L.V., S.J. et al. Analysis of fitting growth models in jinghai mixed-sex yellow chicken. J. Poul. Sci., v.5, p.517-521, 2006. 